\title{
Epidermal stem cell-derived exosomes promote skin regeneration by downregulating transforming growth factor- $\beta 1$ in wound healing
}

Mengna Duan ${ }^{1,2}$, Yan Zhang ${ }^{1}$, Haiyang Zhang ${ }^{1}$, Yupeng Meng ${ }^{1}$, Ming Qian ${ }^{1 *}$ and Guokun Zhang ${ }^{3,4^{*}}$ (D)

\begin{abstract}
Background: Scar formation, which may be caused by myofibroblast aggregations, is the greatest challenge during skin wound healing in the clinical setting. Studies have indicated that epidermal stem cells (EPSC) improve wound healing and reduce scar formation.

Methods: We investigated the therapeutic effects of EPSC-derived exosomes (EPSC-ExOS) on skin wound healing in a skin-defect rat model. We also examined the roles of EPSC-Exos-specific microRNAs in inhibiting the differentiation of human dermal fibroblasts (HDF) into myofibroblasts.

Results: We found that EPSC-Exos increased the wound healing rate and reduced scar formation in rats. Also, EPSCExos improved the regeneration levels of skin appendages, nerves and vessels, as well as the natural distribution of collagen. Furthermore, we found these functions may be achieved by inhibiting the activity of transforming growth factor- $\beta 1$ (TGF- $\beta 1$ ) and its downstream genes. The results showed that some specific microRNAs, including miR-16, let-7a, miR-425-5p and miR-142-3p, were enriched in EPSC-Exos. EPSC-Exos-specific microRNAs, especially miR-425$5 p$ and miR-142-3p, played vital roles in inhibiting myofibroblast differentiation via reducing the TGF- $\beta 1$ expression in dermal fibroblasts.
\end{abstract}

Conclusion: We found a novel function of EPSC-Exos-specific microRNAs, suggesting that EPSC-Exos might represent a strategy to prevent scar formation during wound healing in the clinical setting.

Keywords: Exosome, Epidermal stem cells, MicroRNA, Myofibroblast, Transforming growth factor- $\beta 1$

\section{Background}

The skin is a multilayer interface between the body and the environment, which can regulate temperature, prevent dehydration, keep out pathogens, provide sensation and transport water [1-3]. Once the skin is damaged, the body will immediately start a repair programme to

\footnotetext{
* Correspondence: qianming@jlu.edu.cn; zhang-guokun@hotmail.com 'Department of Prosthodontics, Hospital of Stomatology, Jilin University, 1500 Qinghua Rd., Changchun 130021, Jilin, China

${ }^{3}$ Institute of Antler Science and Product Technology, Changchun Sci-Tech University, 1345 Pudong Rd., Changchun 130600, Jilin, China

Full list of author information is available at the end of the article
}

complete the recovery of the skin's structure and function. However, fibrosis or scarring during the healing process cannot be avoided, even under optimal conditions [3, 4]. Many approaches have attempted to achieve the best healing result, namely the double reconstruction of structure and function. Stem cell-based therapy, with unique advantages, is emerging as a promising candidate for wound healing and skin regeneration [5, 6]. Epidermal stem cells (EPSC) are a type of autologous adult stem cells that are easily obtainable from the skin. EPSC reportedly differentiated into hair follicles and sweat

(c) The Author(s). 2020 Open Access This article is licensed under a Creative Commons Attribution 4.0 International License, which permits use, sharing, adaptation, distribution and reproduction in any medium or format, as long as you give appropriate credit to the original author(s) and the source, provide a link to the Creative Commons licence, and indicate if changes were made. The images or other third party material in this article are included in the article's Creative Commons licence, unless indicated otherwise in a credit line to the material. If material is not included in the article's Creative Commons licence and your intended use is not permitted by statutory regulation or exceeds the permitted use, you will need to obtain permission directly from the copyright holder. To view a copy of this licence, visit http://creativecommons.org/licenses/by/4.0/ The Creative Commons Public Domain Dedication waiver (http://creativecommons.org/publicdomain/zero/1.0/) applies to the data made available in this article, unless otherwise stated in a credit line to the data. 
glands for use in tissue repair, decreasing scar formation and providing long-term regeneration [7-11]. However, there is only a small amount of EPSC in the healing tissue after EPSC treatment. According to the mainstream view [12], the strong paracrine ability of EPSC may be the essential mechanism resulting in these therapeutic effects.

Exosomes, secreted by parental cells, are a type of membrane structure, encapsulating various mRNAs, miRNAs, LncRNAs, CircRNA and proteins to regulate the activity of recipient cells. Exosomes play important roles in tissue regeneration [13-15]. They have a similar function to their parent cells, but they are easier to store and transport, protecting the bioactive substances they carry from adverse conditions such as $\mathrm{pH}$ environments, high temperature and repeated freezing and thawing [16-18]. Moreover, they avoid many of the risks associated with cell transplantation. Therefore, exosome therapy may be a more safe and efficient option.

Transforming growth factor- $\beta 1$ (TGF- $\beta 1$ ) is the primary regulator in wound healing and tissue repair. It is responsible for cellular proliferation, differentiation and metabolism $[3,19]$. Excessive scar formation and fibrosis are closely related to high expression levels of TGF- $\beta 1$ in wound healing $[1,3,20]$. TGF- $\beta 1$ induces the differentiation of myofibroblasts via the phosphorylation of the SMAD family of proteins and activating downstream-related genes such as $\alpha$-smooth muscle actin ( $\alpha$-SMA) and collagen I $[3,19,20]$. Therefore, inhibiting the activities of TGF- $\beta 1$ and its downstream genes might reduce myofibroblast formation and over-aggregation to decrease scarring.

In the current study, we investigated whether EPSCderived exosomes (EPSC-Exos) are effective in the healing of cutaneous wounds using full-thickness skin-defect rat models. Moreover, we identified two specific microRNAs carried by EPSC-Exos as critical components contributing to the suppression of the differentiation of fibroblasts to myofibroblasts by inhibiting TGF- $\beta 1$ expression. Thus, the results suggest that EPSC-Exos treatment could be a potential strategy to prevent excessive fibrosis and scar formation during wound healing in injured skin.

\section{Materials and methods}

\section{Cell culture}

The cell lines of EPSC and human dermal fibroblasts (HDF) were obtained from the China-Japan Union Hospital of Jilin University. Cells were cultured in Dulbecco's modified Eagle medium $(+10 \%$ foetal bovine serum; Biological Industries, Beit-Haemek, Israel) with $5 \% \mathrm{CO}_{2}$ at $37^{\circ} \mathrm{C}$. Cells at the $3-5$ passages were used for this study.

\section{Exosome isolation}

EPSC-Exos were purified by ultracentrifugation according to previously reported methods [15, 18]. Briefly,
EPSC were cultured in a serum-free medium (Gibco, Grand Island, NY) for $48 \mathrm{~h}$. The culture suspension was filtered $(0.1-\mu \mathrm{m}$ filter device) and concentrated $(100-\mathrm{kDa}$ molecular weight cutoff), then loaded onto a $30 \%$ sucrose/deuterium oxide (D2O) cushion and ultracentrifuged at $100,000 \times g$ for $3 \mathrm{~h}$. The exosomes were then washed with phosphate-buffered saline (PBS) 3 times and centrifuged at $1500 \times g$ for $30 \mathrm{~min}(100-\mathrm{kDa}$ molecular weight cutoff). The protein concentration of the exosomes was determined using a bicinchoninic acid protein assay kit (Solarbio, Beijing, China). The exosomes were stored at $-80^{\circ} \mathrm{C}$.

\section{Creation of skin injury model in rats and treatment with EPSC-Exos}

The Animal Experimental Ethics Committee' of Jilin University approved all study protocols and procedures (Approval No. SY201902010). Thirty Sprague Dawley rats (8week-old, female, $200 \mathrm{~g}$ ) were purchased from Liaoning Changsheng Biotechnology Co., Ltd. (Benxi, China) and reared under standard experimental animal feeding conditions. The rats were anaesthetised with $10 \%$ chloral hydrate $(3 \mathrm{ml} / \mathrm{kg})$, after which their hair was removed from the dorsal surface. A 12-mm diameter was removed to establish a full-thickness skin-defect model.

EPSC-Exos was dissolved into HydroMatrix (Sigma-Aldrich, St. Louis, MO) used as a scaffold. Briefly, $100 \mu \mathrm{g} / \mathrm{ml}$ of EPSC-Exos dissolved in PBS and $10 \mathrm{mg} / \mathrm{ml}$ (1\%) hydrogel mixed at a ratio of 1:1 and injected $(200 \mu \mathrm{l})$ around the wound once a week for 4 weeks. Skin damage was recorded photographically every 7 days. The area of each wound was calculated out using Adobe Photoshop CS6. Firstly, lasso tool was used to trace the edge of a wound on a photograph and to circle it; then, we calculate the circled area based on the pixels of that area $(1 \mathrm{~cm}=28.346$ pixels). PBS alone served as a negative control, and epidermal growth factor (EGF; Beyotime, Shanghai, China) mixed with hydro-matrix served as a positive control, both PBS and EGF + hydro-matrix were also injected around the wound once a week for 4 weeks.

The rats were euthanised after being anaesthetised with $10 \%$ chloral hydrate $(3 \mathrm{ml} / \mathrm{kg})$, and the healing wound tissues collected for later use.

\section{Histopathological analysis}

Skin tissue was embedded in paraffin, then sliced into 4$\mu \mathrm{m}$ sections. The sections were stained with haematoxylin and eosin (Solarbio, Beijing, China), Masson (Solarbio), immunohistochemistry (IHC), following the manufacturer's instructions and conventional methods. The primary antibodies used in IHC were anti-CD31 (bs-0468R, 1:500 dilution; Bioss, Beijing, China), anti-Nestin (bs0008R, 1:500 dilution; Bioss) and Ki67 (bs-23105R, 1:500 dilution; Bioss). Sections were photographed using the 
Digital Imaging Scanning System (Precipoint M8; Precipoint, Freising, Germany). The Masson staining and IHC results were analysed using Image-Pro Plus software. Firstly, we counted the area of the target item in the picture and then calculated its ratio to the total area of the picture.

\section{Co-culture system of EPSC and HDF}

The $5 \times 10^{4} \mathrm{HDF}$ and ESCs were seeded with Dulbecco's modified Eagle medium and 10\% foetal bovine serum on the upper $(0.4 \mu \mathrm{m})$ and lower chambers of 24-well plates (Corning Life Sciences, NY). After incubating plates for $48 \mathrm{~h}$ at $37^{\circ} \mathrm{C}$, TGF- $\beta 1$ expression levels of HDF were performed using quantitative immunofluorescence (IF) staining and quantitative real-time polymerase chain reaction (qRT-PCR).

\section{qRT-PCR}

Trizol reagent (Tiangen, Beijing, China) was used for total RNA isolation of the skin tissue. cDNA was prepared via reverse transcription of RNA. CDNA, primers, and SYBR premix (Roche, Basel, Switzerland) were combined on qRT-PCR using the ABI 9700 Detection System (Thermo Fisher Scientific, Waltham, MA), and GAPDH was used as control. Supplemental Table S1 shows the primers. All experiments were repeated three times. qRT-PCR for miRNA was done using miScript SYBR Green PCR kit (Qiagen, Hiden, Germany) according to the manufacturer's instruction. All results were normalised to U6 small RNA levels measured using the Hs_RNU6B_2 miScript Primer Assay kit (Qiagen). Supplemental Table S1 shows the primers. All experiments were repeated three times.

\section{IF staining}

HDF with a density of $70-80 \%$ were incubated for 10 min with $10 \%$ formaldehyde and for $30 \mathrm{~min}$ with $1 \%$ bovine serum albumin (Beyond, Shanghai, China). Next, the cells were incubated with anti-TGF- $\beta 1$ (BSM$33345 \mathrm{M}, 1: 500$ dilution; Bioss) at $4{ }^{\circ} \mathrm{C}$ overnight and incubated with anti-IgG (A0408, 1:500 dilution) at $25^{\circ} \mathrm{C}$ for $30 \mathrm{~min}$. The nucleus was labelled with 4',6-diamidino-2-phenylindole (Beyotime, Shanghai, China).

\section{Statistical analysis}

All quantitative data were expressed as the mean \pm SD . One-way analysis of variance with Tukey's multiple comparisons test was used for statistically significant
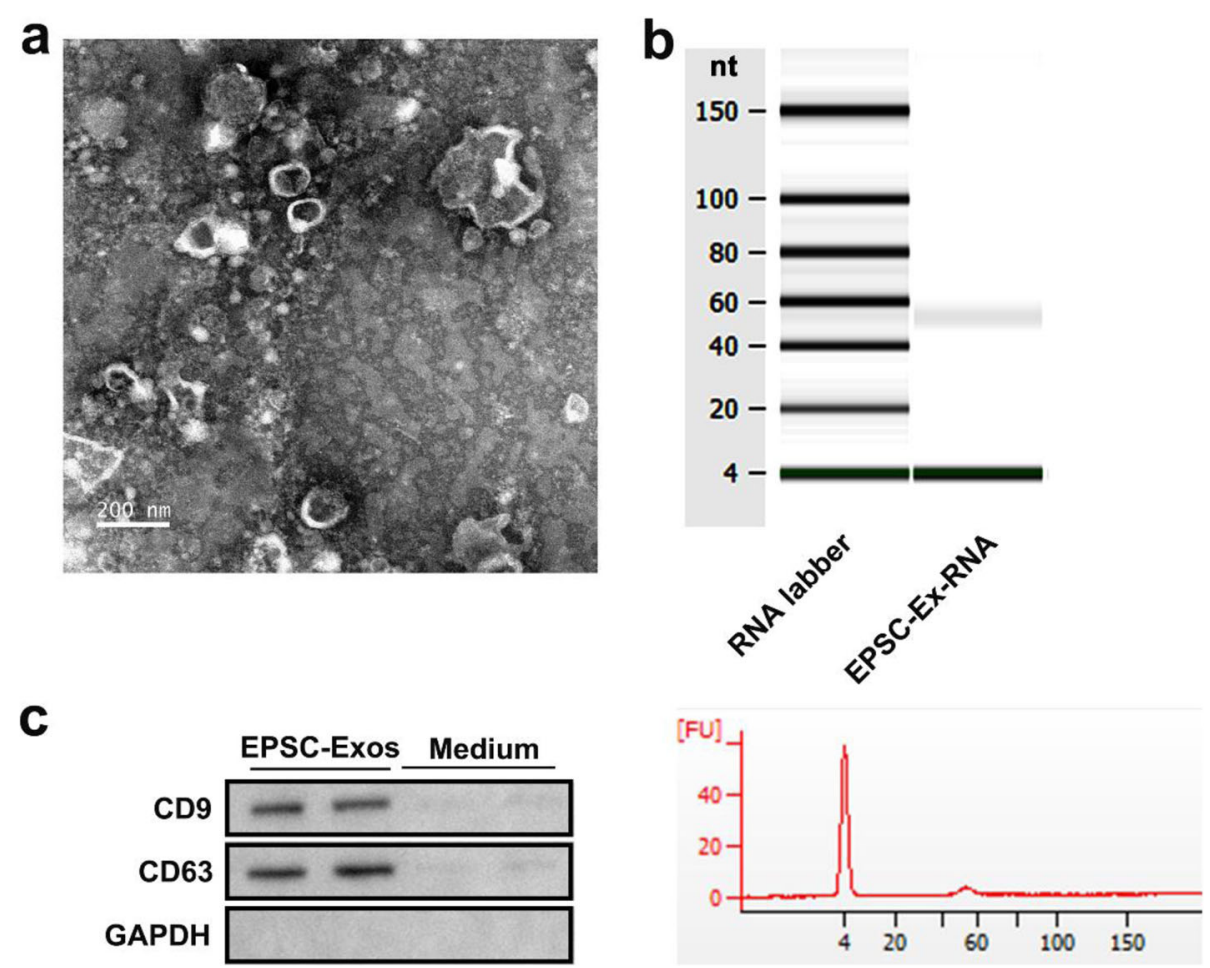

Fig. 1 Characterisation of exosomes derived from EPSC. EPSC-Exos were isolated and purified from EPSC supernatant using high-speed centrifugation. a Identification of the main morphological characteristics of EPSC-Exos by transmission electron microscopy images. Scale bar = $100 \mathrm{~nm}$. b RNA expression profiles of EPSC-Exos by Genechip analysis. c The markers of exosomes are detected using western blot assay, and the cell-free medium was added as a control. EGF, epidermal stem cells; EPSC-Exos, epidermal stem cell-derived exosomes 
differences. Results were considered significant at a $P$ value of less than 0.05 .

\section{Results}

EPSC-Exos improve wound healing rate and suppress scar formation

We first identified the purified EPSC-Exos. Transmission electron microscopy results showed that the particle size of EPSC-Exos was between 30 and $100 \mathrm{~nm}$ (Fig. 1a). The RNA in EPSC-Exos was analysed via agarose gel electrophoresis. There was only a clear band at $60 \mathrm{nt}$ (Fig. 1b). Analysis using the Agilent 2100 Bioanalyzer confirmed these results (Fig. 1b). Western blot analysis was used to detect the expression of exosomal markers CD9 and CD63, and serum-free medium was used as a control. The results showed that CD9 and CD63 were abundant in EPSC-Exos (Fig. 1c). In summary, the isolated EPSC-Exos has high purity and can be used in subsequent experiments.

To clarify the effects of EPSC-Exos in the wound healing rate and scar formation, we used full-thickness skin-defect rats and injected equal quantities of hydrogel-coated EPSC-Exos, PBS or EGF around the wounds. The results are shown in Fig. 2. After 1 or 2 weeks of treatment, the wound areas and scars of the EPSC-Exos group were smaller than those of the EGF and control groups. After 3 weeks, the defects in the EPSC-Exos group were closed,

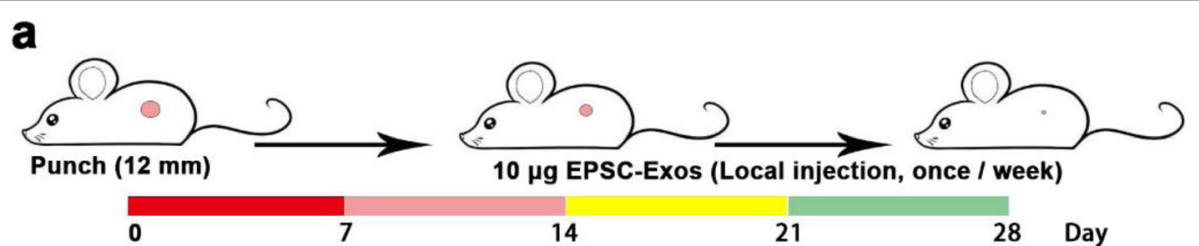

b
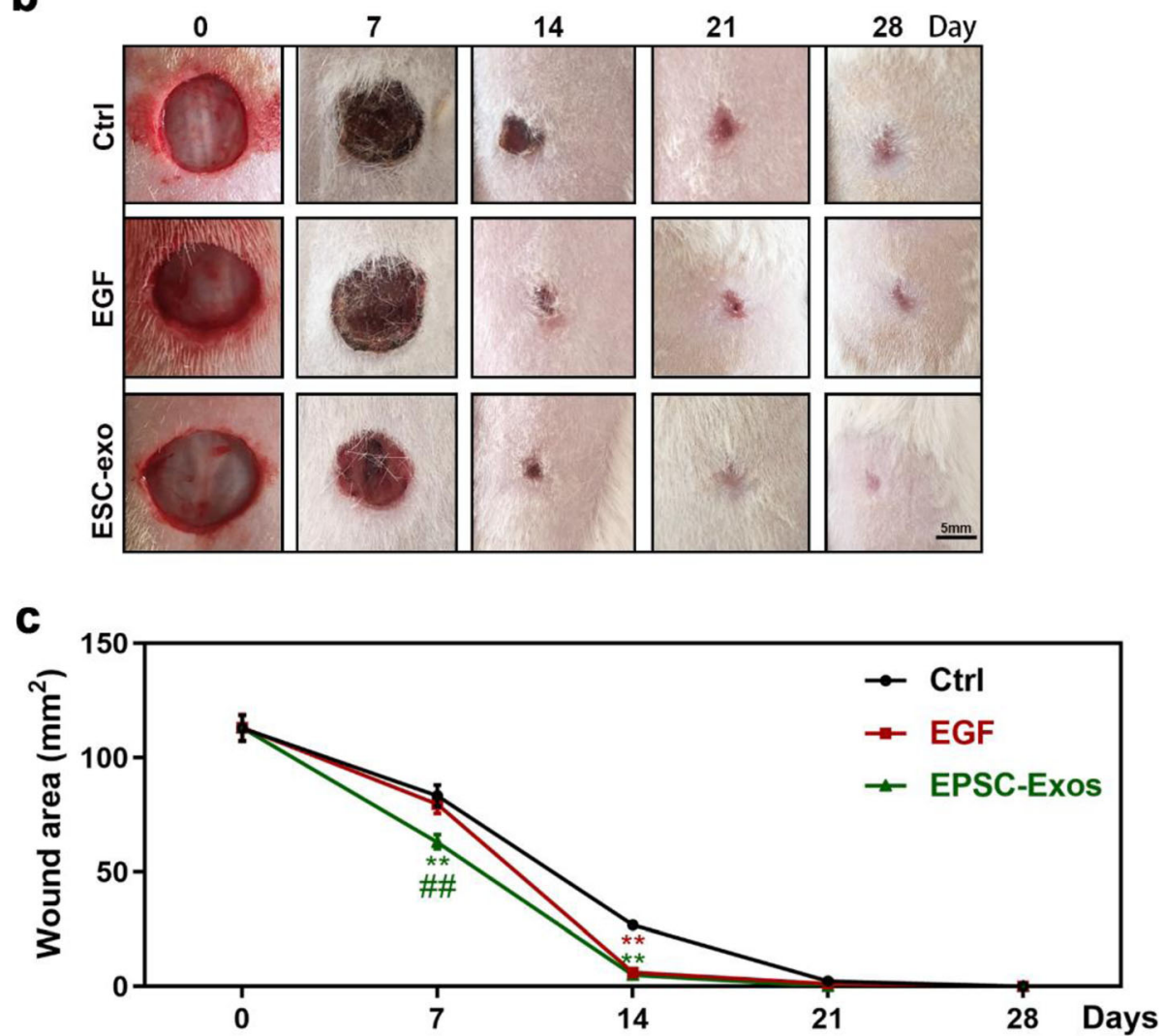

Fig. 2 EPSC-Exos increased the wound healing rate and suppressed scar formation in a full-thickness skin-defect rat model. a Experimental procedure. The dorsal area of the rats was shaved under anaesthesia. Then, circular hole (12-mm diameter), full-thickness skin excisional wounds were made on the shaved skin. The rats were randomly divided into three groups ( $n=10 /$ group): phosphate-buffered saline (PBS, negative control), epidermal growth factor (EGF, positive control, $10 \mu \mathrm{g}$ ), and EPSC-Exos (positive control; $10 \mu \mathrm{g}$ ). The rats were treated via local injection around the wound once a week for 4 weeks, and skin damage was recorded photographically every 7 days. $\mathbf{b}$ Overall morphological changes observed during the wound healing. Scale bar $=5 \mathrm{~mm}$. c Changes in wound area during healing. ${ }^{* *} P<0.01$ compared with the control; ${ }^{\# \#} P<0.01$ compared with EGF; mean \pm SD. Ctrl, control; EGF, epidermal growth factor; EPSC-Exos, epidermal stem cell-derived exosomes 
but those in the EGF and control groups were not. After 4 weeks, the defects in the EGF and control groups were also closed. However, they exhibited more scar formation than that observed in the EPSC-Exos group. These results suggest that EPSC-Exos can improve the wound healing rate and suppress scar formation.

\section{EPSC-Exos improve the regeneration level of skin} appendages and collagen distribution on wound healing of rats

After evaluating the effect of EPSC-Exos on increasing the wound healing rate and decreasing scar formation in shape and appearance, we made further evaluations at the histological level. As shown in Fig. 3a and b, the results showed that after 4 weeks of treatment, the skin in the EPSC-Exos group exhibited more appendages than that in the EGF and control groups (significant; $P<$ 0.001 ). There were significantly fewer myofibers (red) in the EPSC-Exos group than the EGF and control groups $(P<0.001)$ (Fig. 3a, c). In addition, there were significantly fewer collagen fibres (blue) in the skin of the EPSC-Exos group than observed in the EGF group $(P<$ 0.01) (Fig. 3a, d). Moreover, among the regenerated collagen fibres, the rates of collagen I and III in the EPSC-

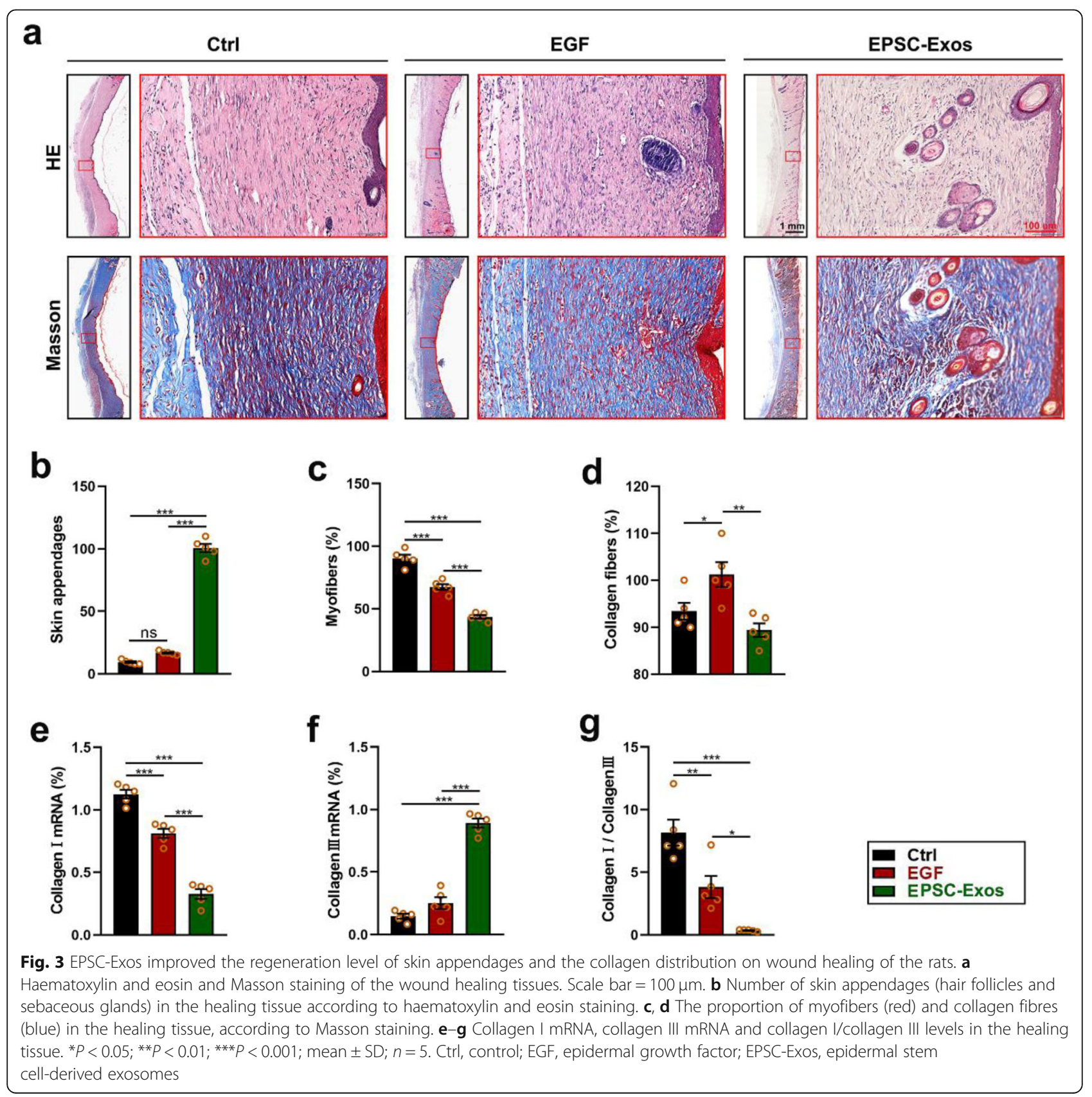


Exos group were lower than in the EGF and control groups (Fig. 3e-g). These results suggest that EPSCExos can promote skin appendage regeneration, decrease myofiber formation and increase collagen III in wound healing.

\section{EPSC-Exos promote nerve and vessel regeneration on wound healing in rats}

Next, we clarified the effects of EPSC-Exos on nerve and vessel regeneration of damaged skin using IHC. The results showed that after 4 weeks of treatment (as shown in Fig. $4 \mathrm{a}-\mathrm{c}$ ), the skin of the EPSC-Exos group exhibited significantly more vessels $\left(\mathrm{CD} 31^{+}\right)$and nerves $\left(\right.$nestin $\left.^{+}\right)$ than in the EGF and control groups $(P<0.001)$. Also, the proliferation $(\mathrm{Ki} 67+)$ of skin cells in the EPSC-Exos group was also higher than in the EGF and control groups (Fig. 4d). These results suggest that EPSC-Exos can promote nerve and vessel regeneration and cell proliferation in wound healing.

\section{EPSC-Exos inhibit TGF- $\beta 1$ expression from suppressing myofibroblast formation in vivo and in vitro}

TGF- $\beta 1$ is the main factor in inducing dermal fibroblasts and the most important cell in skin wound healing and for differentiation into myofibroblasts [1, 3, 4]. Inhibiting TGF- $\beta 1$ may be an essential means to reduce scar formation in wound healing. To explore the mechanism of EPSC-Exos inhibiting scar formation in wound healing, we measured the TGF- $\beta 1$ expression levels in the healing tissue of rat models and HDF co-cultured with ESCs. The results showed that the mRNA levels of TGF- $\beta 1$ and its downstream genes, including Smad2, $\alpha$-SMA and collagen $I$, in the healing tissue of rats were all significantly lower than in the EGF and control groups $(P<$

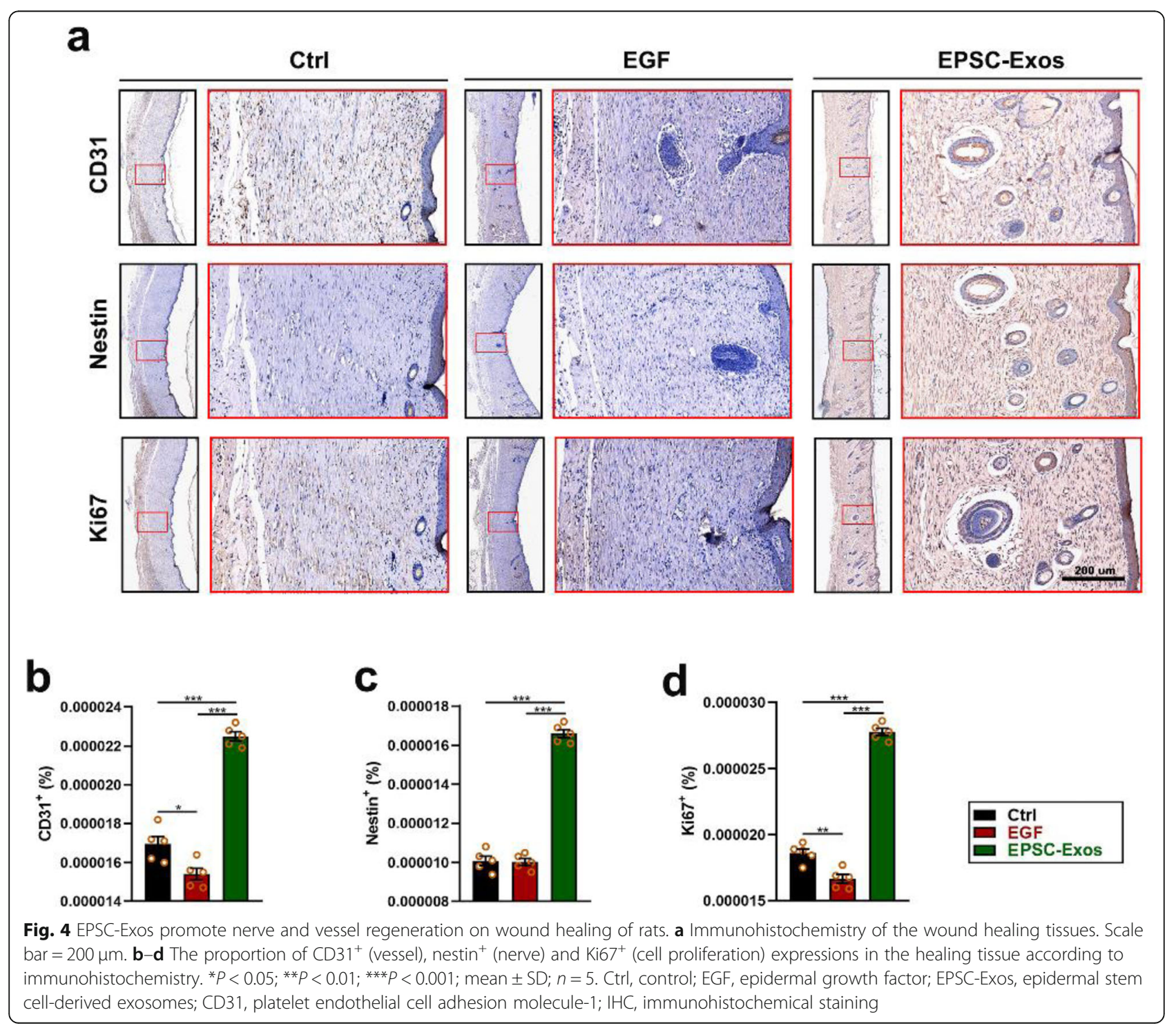


0.001) (Fig. 5a-d). Also, expression levels of TGF- $\beta 1$ were evaluated on HDF treated with EPSC-Exos, cocultured with ESC in Transwell plates for $48 \mathrm{~h}$ (Fig. 5e). As shown in Fig. $5 \mathrm{f}$ and $\mathrm{g}$, expression levels of TGF- $\beta 1$ treated with EPSC-Exos were significantly reduced in mRNA and protein compared with the control group $(P<0.001)$. These results suggest that EPSC-Exos may inhibit the expression of TGF- $\beta 1$ to suppress myofibroblast formation in wound healing.

\section{EPSC-Exos-specific microRNAs target TGF- $\beta 1$ to suppress myofibroblast differentiation}

We found that miR-16, let-7a, miR-425-5p and miR142-3p were highly expressed in EPSC-Exos detected using qRT-PCR (Fig. 6a). To predict the potential target genes of these microRNAs, we used TargetScan (http:// www.targetscan.org/) and Microrna (http://www.microrna.org) for analysis. The results showed that miR-425$5 p$ and miR-142-3p were all directly targeted to TGF- $\beta 1$ (Fig. 6b) To verify the role of miR-425-5p and miR-142$3 p$ in EPSC-Exos, we first added agomirs in HDF cultural system to test whether they can affect the expression of TGF- $\beta 1$. The qRT-PCR and IF analysis showed that these 2 microRNAs significantly suppressed the expression of TGF- $\beta 1$ (Fig. $6 \mathrm{c}, \mathrm{d}$ ). The results suggest that EPSC-Exos may inhibit the differentiation of fibroblasts to myofibroblasts via suppressing TGF- $\beta 1$ expression via miR-425-5p and miR-142-3p.

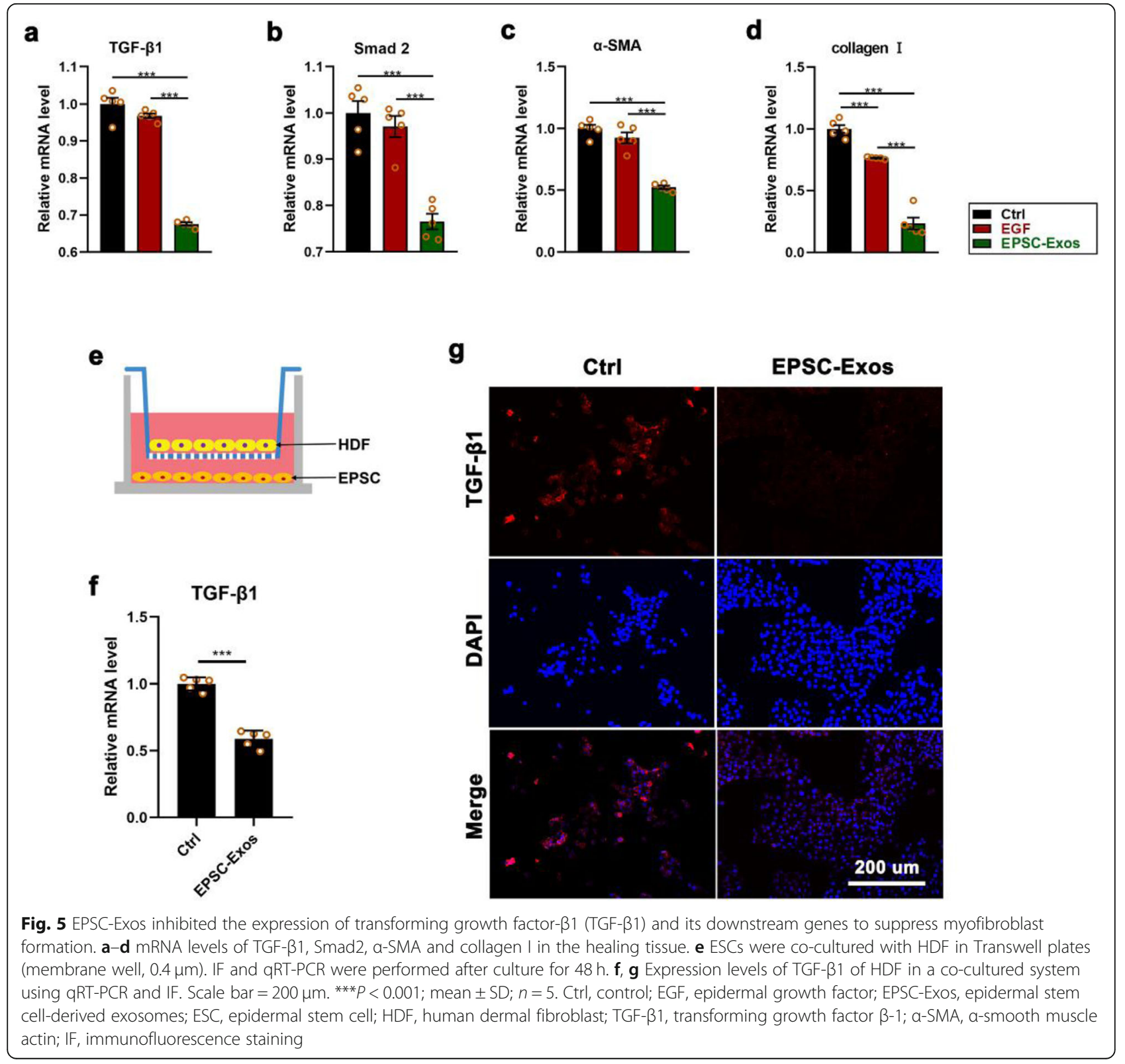




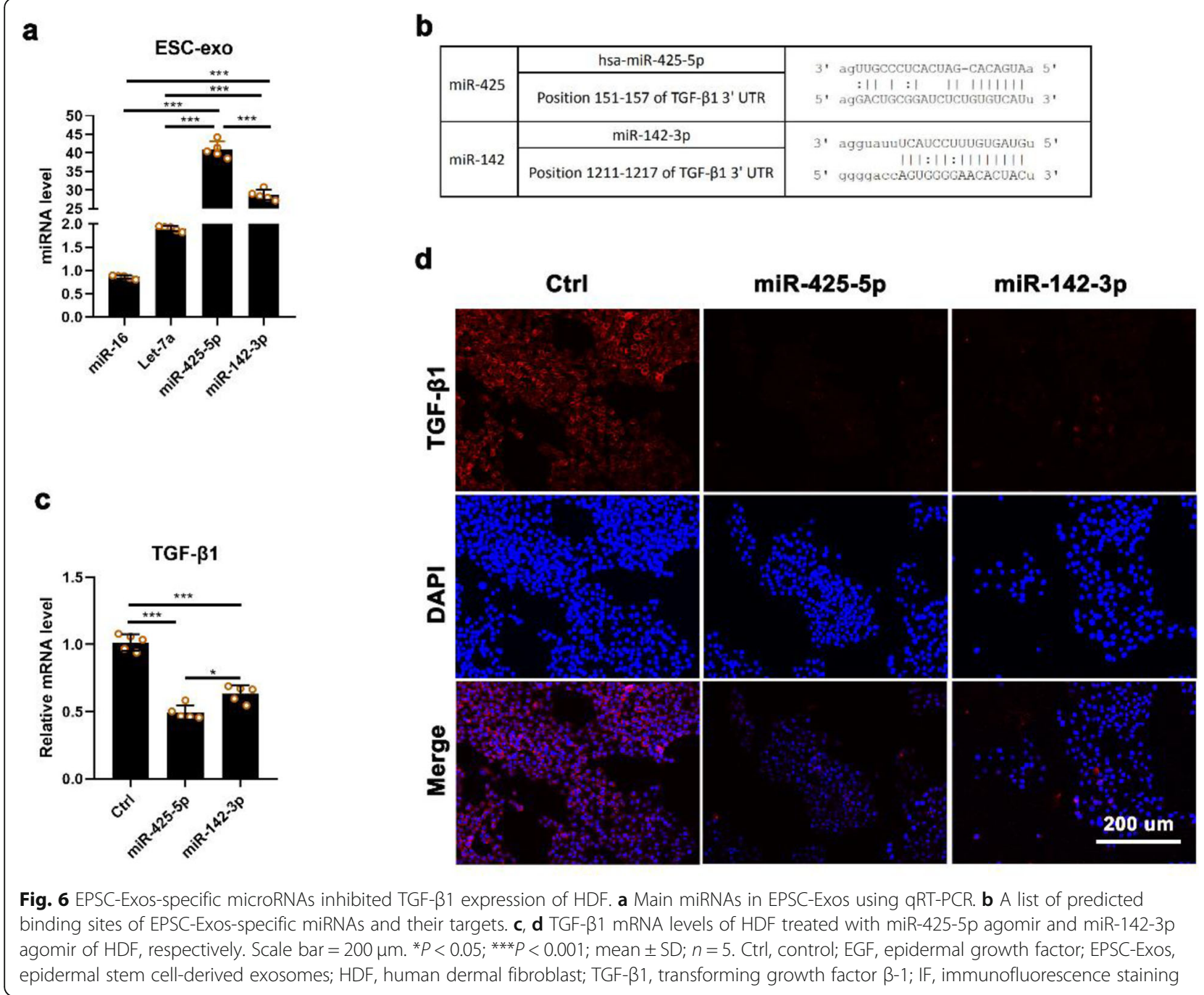

\section{Discussion}

Scar formation is an undesirable and significant result of both wound healing and fibrosing disorders. Excessive accumulation of myofibroblasts is a crucial factor for scar development, which can even lead to tissue or organ contraction [1, 4, 21]. Regulating myofibroblast formation may be an effective approach to preventing abnormal scarring. EPSC-based therapies have been proven to improve wound healing and reduce scar formation [22, 23]. Researchers have evaluated the effects of EPSC on tissue repair and wound healing. However, no previous studies have focused on the effects of EPSC-Exos on wound healing and scar formation. This study is the first to report that EPSCExos suppress myofibroblast differentiation in wound healing, which may be achieved by EPSC-Exosspecific microRNAs. Our research provides new insights for the application of EPSC-Exos to prevent scar formation.
EPSC, similar to adipose stem cells, are a cell type widely used in clinical research and regenerative medicine because they are numerous, are easy to obtain and have fewer ethical controversies than embryonic stem cells [22, 24, 25]. EPSC promote re-epithelisation, angiogenesis and hair growth in wound healing, as well as stimulate the recruitment and proliferation of endogenous stem cells $[22,23,25]$. We speculated that EPSC might play a major role in cell therapy through paracrine. Therefore, the paracrine functions of EPSC were the focus of this study. We observed that the healing skin in the EPSC-Exos group was smooth with less scarring, which was significantly lower than that in the control and EGF groups.

Furthermore, we confirmed that the expression levels of TGF- $\beta 1$ were lower than in the control and EGF groups. TGF- $\beta 1$ is reportedly a pivotal factor in simulating fibroblast differentiation into myofibroblasts $[26,27]$. Therefore, intervention is required during wound 


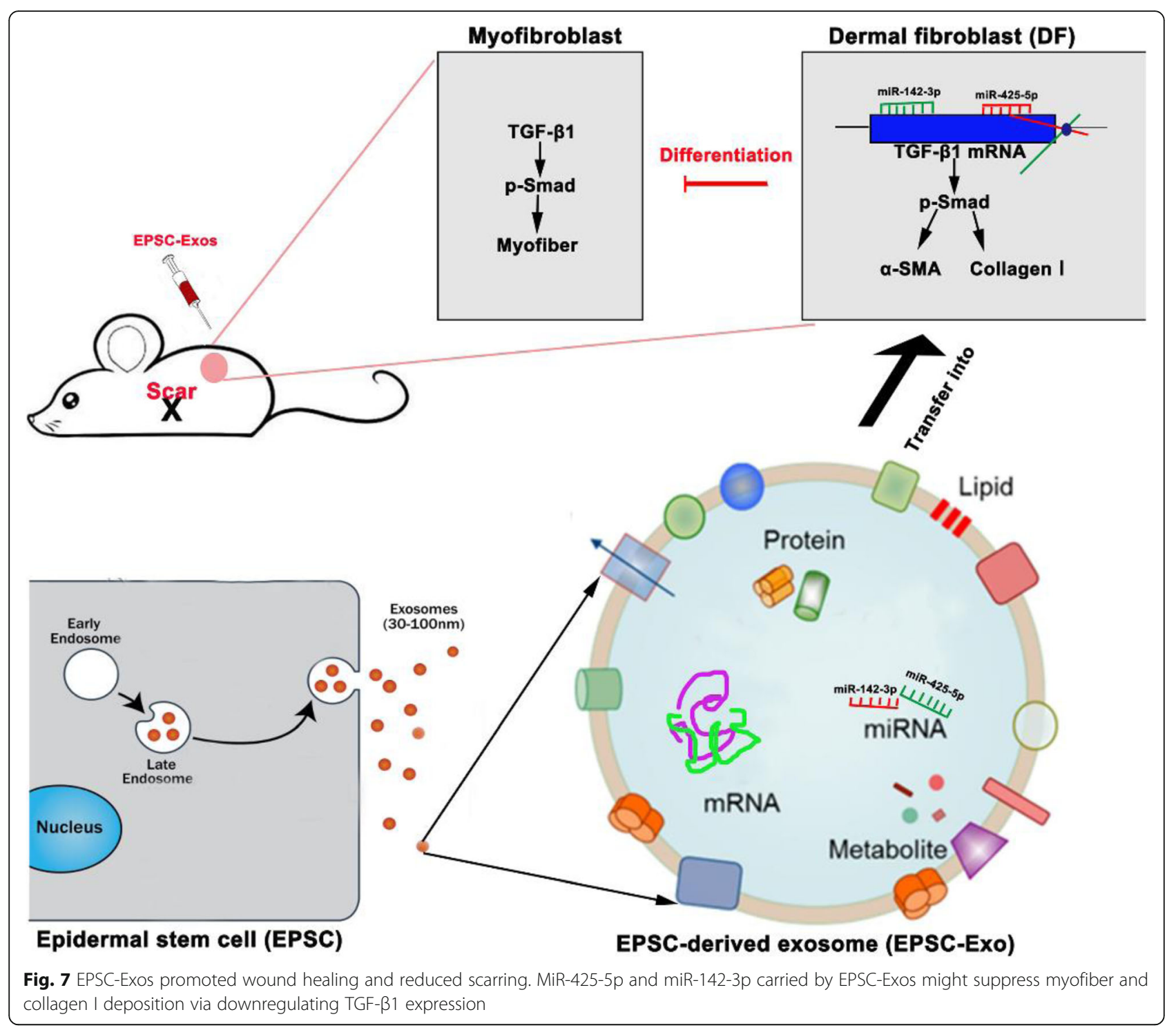

healing to prevent excessive formation of myofibroblasts, rather than taking remedial measures after scar formation.

Exosomes have content that includes mRNAs, regulatory miRNAs, functional protein and signalling lipids. Thus, they involve cell signalling and cell-to-cell communication and they influence tissue responses to injury, infection and disease [14, 28]. In this study, we found that the primary function of promoting skin healing and reducing scar formation may be caused by EPSC-Exosspecific miRNAs. The EPSC-Exos-specific miRNAs inhibited myofibroblast formation, which might be related to the targeting of TGF- $\beta 1$-targeting microRNAs. In our previous study, several highly expressed specific microRNAs derived from EPSC-Exos were identified, including miR-16, let-7a, miR-425-5p and miR-142-3p. miR-425-5p [29] and miR-142-3p [30] have been previously reported to suppress fibrotic diseases, and most studies have suggested that these two microRNAs directly target TGF- $\beta 1$, consistent with our present findings. We believe the functions of microRNAs in different organs and tissues might be the same. Therefore, our study on the effects of EPSC-Exos on these two microRNAs might help us better understand microRNAs' functions. We believe these EPSC-Exos miRNAs could be essential inhibitors of TGF- $\beta 1$, reducing the myofibroblast formation during skin wound healing.

Exosome-derived microRNAs are more stable than their parental cells. They can resist degradation in the circulation process in the body through the vesicle structure released by the parental cells $[18,31]$. In the subsequent production and application, we can use EPSC as a factory to produce exosomes, making EPSC produce more functional microRNAs via transfection. Compared with EPSC, 
EPSC-Exos might be safer and more efficient in clinical applications. They may also have other advantages, such as easier storage and production, a lower risk of side effects and easier quality control. Thus, we suggest that EPSC-Exos could be a candidate strategy for promoting healing and reducing scar formation in the future.

\section{Conclusions}

This study has shed light on the specific microRNAs of EPSC-Exos and clarified a new approach for using stem cell therapy to promote wound healing and reduce scarring. miR425-5p and miR-142-3p from EPSC-Exos might suppress myofiber and collagen I deposition via downregulating TGF$\beta 1$ expression (Fig. 7). As an alternative to cell therapy, EPSCExos might have a clinically beneficial anti-scarring effect.

\section{Supplementary information}

Supplementary information accompanies this paper at https://doi.org/10. 1186/s13287-020-01971-6.

\section{Additional file 1.}

\section{Abbreviations}

a-SMA: a-Smooth muscle actin; CD31: Platelet endothelial cell adhesion molecule-1; EGF: Epidermal growth factor; EPSC: Epidermal stem cells; EPSCExos: EPSC-derived exosomes; HDF: Human dermal fibroblasts; IF: Immunofluorescence staining; IHC: Immunohistochemical staining; PBS: Phosphate-buffered saline; TGF- $\beta 1$ : Transforming growth factor- $\beta 1$

\section{Acknowledgements}

We thank Professor Wang from the China-Japan Union Hospital of Jilin University for providing the epidermal stem cells and human dermal fibroblasts.

\section{Authors' contributions}

$M D$ and $G Z$ designed and supervised the research; $Y Z$ performed the research and analysed the data; $\mathrm{HZ}$ and $\mathrm{MQ}$ contributed to animal experiments; MD and YM contributed to histopathological experiments; and MD, MQ and GZ wrote and edited the paper. All authors read and approved the final manuscript.

\section{Funding}

This work was supported by the Jilin Provincial Department of Education Science and Technology Program (China, No. JJKH20190104KJ) and 2020 Science and Technology Project of Jilin Provincial Finance Department (China, No. jcsz2020304-19).

\section{Availability of data and materials}

The datasets used and/or analysed during the present study are available from the corresponding author on reasonable request.

\section{Ethics approval and consent to participate}

This study was approved by the Administration Committee of Experimental Animals Jilin University (Approval No. SY201902010).

\section{Consent for publication}

Not applicable.

\section{Competing interests}

The authors declare that they have no competing interests.

\section{Author details}

${ }^{1}$ Department of Prosthodontics, Hospital of Stomatology, Jilin University, 1500 Qinghua Rd., Changchun 130021, Jilin, China. ${ }^{2}$ Jilin Provincial Laboratory of Biomedical Engineering, Jilin University, 1500 Qinghua Rd., Changchun 130021, Jilin, China. ${ }^{3}$ Institute of Antler Science and Product
Technology, Changchun Sci-Tech University, 1345 Pudong Rd., Changchun 130600, Jilin, China. ${ }^{4}$ Institute of Special Animal and Plant Sciences, Chinese Academy of Agricultural Sciences (CAAS), 4899 Juye St., Changchun 130112, Jilin, China.

Received: 24 August 2020 Accepted: 11 October 2020

Published online: 23 October 2020

\section{References}

1. L. Gravitz, Skin, Nature, 563 (2018) S83.

2. A.V. Nguyen, A.M. Soulika, The dynamics of the skin's immune system, Int J Molecular Sci 201920

3. Martin P. Wound healing--aiming for perfect skin regeneration. Science (New York, N.Y.). 1997;276:75-81.

4. A.L. Rippa, E.P. Kalabusheva, E.A. Vorotelyak, Regeneration of dermis: scarring and cells involved. Cells 20198.

5. Lee DE, Ayoub N, Agrawal DK. Mesenchymal stem cells and cutaneous wound healing: novel methods to increase cell delivery and therapeutic efficacy. Stem Cell Res Ther. 2016;7:37.

6. Hassan WU, Greiser U, Wang W. Role of adipose-derived stem cells in wound healing. Wound Repair Regeneration. 2014;22:313-25.

7. Dunnwald M, Tomanek-Chalkley A, Alexandrunas D, Fishbaugh J, Bickenbach JR. Isolating a pure population of epidermal stem cells for use in tissue engineering. Exp Dermatol. 2001:10:45-54.

8. Redvers RP, Li A, Kaur P. Side population in adult murine epidermis exhibits phenotypic and functional characteristics of keratinocyte stem cells. Proc Natl Acad Sci U S A. 2006;103:13168-73.

9. Schlüter H, Paquet-Fifield S, Gangatirkar P, Li J, Kaur P. Functional characterization of quiescent keratinocyte stem cells and their progeny reveals a hierarchical organization in human skin epidermis. Stem cells (Dayton, Ohio). 2011;29:1256-68.

10. Pellegrini G, Ranno R, Stracuzzi G, Bondanza S, Guerra L, Zambruno G, Micali G, De Luca M. The control of epidermal stem cells (holoclones) in the treatment of massive full-thickness burns with autologous keratinocytes cultured on fibrin. Transplantation. 1999:68:868-79.

11. Walmsley GG, Maan ZN, Wong WW, Duscher D, Hu MS, Zielins ER, Wearda T, Muhonen E, McArdle A, Tevlin R, Atashroo DA, Senarath-Yapa K, Lorenz HP, Gurtner GC, Longaker MT. Scarless wound healing: chasing the holy grail. Plast Reconstr Surg. 2015;135:907-17.

12. Wu P, Zhang B, Shi H, Qian H, Xu W. MSC-exosome: a novel cell-free therapy for cutaneous regeneration. Cytotherapy. 2018;20:291-301.

13. Basu J, Ludlow JW. Exosomes for repair, regeneration and rejuvenation. Expert Opin Biol Ther. 2016;16:489-506.

14. Phinney DG, Pittenger MF. Concise review: MSC-derived exosomes for cellfree therapy. Stem cells (Dayton, Ohio). 2017;35:851-8.

15. Goodarzi P, Larijani B, Alavi-Moghadam S, Tayanloo-Beik A, MohamadiJahani F, Ranjbaran N, Payab M, Falahzadeh K, Mousavi M, Arjmand B. Mesenchymal stem cells-derived exosomes for wound regeneration. Adv Exp Med Biol. 2018;1119:119-31.

16. Schepers $\mathrm{K}$, Campbell TB, Passegué E. Normal and leukemic stem cell niches: insights and therapeutic opportunities. Cell Stem Cell. 2015;16:254-67.

17. Anthony BA, Link DC. Regulation of hematopoietic stem cells by bone marrow stromal cells. Trends Immunol. 2014:35:32-7.

18. Fang $S$, Xu C, Zhang Y, Xue C, Yang C, Bi H, Qian X, Wu M, Ji K, Zhao Y, Wang $Y$, Liu $H$, Xing $X$. Umbilical cord-derived mesenchymal stem cellderived exosomal MicroRNAs suppress myofibroblast differentiation by inhibiting the transforming growth factor-B/SMAD2 pathway during wound healing. Stem Cells Transl Med. 2016;5:1425-39.

19. K.K. Kim, D. Sheppard, H.A. Chapman, TGF-B1 signaling and tissue fibrosis, Cold Spring Harbor Perspectives Biology 201810.

20. Lichtman MK, Otero-Vinas M, Falanga V. Transforming growth factor beta (TGF- $\beta$ ) isoforms in wound healing and fibrosis. Wound Repair and Regeneration. 2016;24:215-22.

21. Ehrlich HP. A snapshot of direct cell-cell communications in wound healing and scarring. Advances Wound Care. 2013;2:113-21.

22. Yang $R$, Liu F, Wang J, Chen $X$, Xie J, Xiong $K$. Epidermal stem cells in wound healing and their clinical applications. Stem Cell Res Ther. 2019; 10:229.

23. Pikuła M, Langa P, Kosikowska P, Trzonkowski P. Stem cells and growth factors in wound healing. Postepy higieny i medycyny doswiadczalnej (Online). 2015;69:874-85. 
24. Mesa KR, Kawaguchi K, Cockburn K, Gonzalez D, Boucher J, Xin T, Klein AM, Greco V. Homeostatic epidermal stem cell self-renewal is driven by local differentiation. Cell Stem Cell. 2018;23:677-86 e674.

25. Jackson CJ, Tønseth KA, Utheim TP. Cultured epidermal stem cells in regenerative medicine. Stem Cell Res Ther. 2017;8:155.

26. Ko UH, Choi J, Choung J, Moon S, Shin JH. Physicochemically tuned myofibroblasts for wound healing strategy. Sci Rep. 2019;9:16070.

27. Vallée A, Lecarpentier $Y$. TGF- $\beta$ in fibrosis by acting as a conductor for contractile properties of myofibroblasts. Cell \& bioscience. 2019;9:98.

28. Pegtel DM, Gould SJ. Exosomes. Annu Rev Biochem. 2019;88:487-514.

29. Wang L, Liu J, Xie W, Li G, Yao L, Zhang R, Xu B. miR-425 reduction causes aberrant proliferation and collagen synthesis through modulating TGF- $\beta$ / Smad signaling in acute respiratory distress syndrome. Int J Clin Exp Pathol. 2019;12:2604-12

30. Yang X, Dan X, Men R, Ma L, Wen M, Peng Y, Yang L. MiR-142-3p blocks TGF- $\beta$-induced activation of hepatic stellate cells through targeting TGF $\beta R$ I. Life Sci. 2017;187:22-30.

31. Keller S, Ridinger J, Rupp AK, Janssen JW, Altevogt P. Body fluid derived exosomes as a novel template for clinical diagnostics. J Transl Med. 2011;9:86.

\section{Publisher's Note}

Springer Nature remains neutral with regard to jurisdictional claims in published maps and institutional affiliations.

Ready to submit your research? Choose BMC and benefit from:

- fast, convenient online submission

- thorough peer review by experienced researchers in your field

- rapid publication on acceptance

- support for research data, including large and complex data types

- gold Open Access which fosters wider collaboration and increased citations

- maximum visibility for your research: over $100 \mathrm{M}$ website views per year

At $\mathrm{BMC}$, research is always in progress.

Learn more biomedcentral.com/submissions 